\title{
Simulation Devices in Interventional Radiology: Caveat Emptor
}

Derek A. Gould, ${ }^{1}$ Jim A. Reekers, ${ }^{2}$ David O. Kessel, ${ }^{3}$ Nick C. Chalmers, ${ }^{4}$ Marc Sapoval, ${ }^{5}$ Aalpen A. Patel, ${ }^{6}$ Gary J. Becker, ${ }^{7,8}$ Mick J. Lee, ${ }^{9}$ Luc Stockx ${ }^{10}$

${ }^{1}$ Department of Radiology, Royal Liverpool University Trust, Prescott Street, Liverpool L7 8XP, United Kingdom

${ }^{2}$ Department of Radiology, Academic Medical Centre, Meibergdreef 9, 1105 Amsterdam, The Netherlands

${ }^{3}$ St. James's University Hospital, Leeds Teaching Hospitals, Beckett Street, Leeds LS7 9TF, United Kingdom

${ }^{4}$ Department of Radiology, Manchester Royal Infirmary, Oxford Road, Manchester M13 9WL, United Kingdom

${ }^{5}$ Department of Cardiovascular Radiology, Hôpital Européen Georges Pompidou, 20 Rue Leblanc, 75908 Paris Cedex 15, France

${ }^{6}$ Hospital of the University of Pennsylvania, Department of Radiology, 3400 Spruce Street, 1 Silverstein, Philadelphia, PA 19104, USA

${ }^{7}$ University of Arizona College of Medicine, Department of Radiology, Room 1363, 1501 N. Campbell Ave., Tucson, AZ 85724-5067, USA

${ }^{8}$ Associate Executive Director, Diagnostic Radiology, The American Board of Radiology, 5441 E. Williams Blvd., Suite 200, Tucson, AZ 85711, USA

${ }^{9}$ Department of Radiology, Beaumont Hospital and Royal College of Surgeons in Ireland, Beaumont Road, Dublin 9, Ireland

${ }^{10}$ Department of Medical Imaging, ZOL Campus Sint-Jan, Schiepse Bos 6, 3600 Genk, Belgium

RE: Cardiovasc Intervent Radiol (2006) 29: 4-6 - The following title-page footnote was mistakenly omitted from this CIRSE Guidelines article: This article also appears in the February 2006 issue of Journal of Vascular and Interventional Radiology. 